\title{
ARTICLE Virtual memory CD8 T cells expanded by helminth infection confer broad protection against bacterial infection
}

\author{
J. S. Lin ${ }^{1}$, K. Mohrs ${ }^{1,2}$, F. M. Szaba ${ }^{1}$, L. W. Kummer ${ }^{1}$, E. A. Leadbetter ${ }^{1,3}$ and M. Mohrs ${ }^{1,2}$
}

\begin{abstract}
Epidemiological data and animal studies suggest that helminth infection exerts potent immunomodulatory effects that dampen host immunity against unrelated pathogens. Despite this notion, we unexpectedly discovered that prior helminth infection resulted in enhanced protection against subsequent systemic and enteric bacterial infection. A population of virtual memory CD8 T (CD8 $\mathrm{T}_{\mathrm{VM}}$ ) cells underwent marked expansion upon infection with the helminth Heligmosomoides polygurus by an IL-4-regulated, antigenindependent mechanism. CD8 $\mathrm{T}_{\mathrm{VM}}$ cells disseminated to secondary lymphoid organs and established a major population of the systemic CD8 T cell pool. IL-4 production elicited by protein immunization or selective activation of natural killer T cells also results in the expansion of CD8 $T_{V M}$ cells. Notably, CD8 $T_{V M}$ cells expanded by helminth infection are sufficient to transfer innate noncognate protection against bacteria to naïve animals. This innate non-cognate "collateral protection" mediated by CD8 $T_{V M}$ might provide parasitized animals an advantage against subsequent unrelated infections, and represents a potential novel strategy for vaccination.
\end{abstract}

Mucosal Immunology (2019) 12:258-264; https://doi.org/10.1038/s41385-018-0100-x

\section{INTRODUCTION}

The vast majority of infection studies is conducted in specific pathogen-free laboratory animals that are challenged with individual pathogens. In striking contrast, a history of infection with multiple pathogens is common in humans, especially in developing countries. ${ }^{1}$ Concurrent infections elicit complex immune responses which can potentially interfere with host immunity to secondary pathogen challenge. ${ }^{1-9}$ This consideration is particularly relevant for chronic infections such as helminthiases, which elicit potent immune responses with lasting effects on the immune status. ${ }^{8-11}$ While helminth parasites typically establish chronic infection, they are generally tolerated with limited immunopathology, presumably owing to potent immunomodulatory effects. ${ }^{11,12}$ However, as a negative consequence of immunomodulation, parasitized individuals are generally considered to be more susceptible to secondary infection. ${ }^{1-7}$

Infection with helminth parasites elicits robust Th2-polarized CD4 T cell responses with IL-4 as a critical effector cytokine. ${ }^{11,13}$ In contrast to CD4 T cells, CD8 T cells have no apparent impact on the immunological or parasitological parameters. ${ }^{14}$ Consequently, few studies have analyzed CD8 T cell responses to helminth infection. ${ }^{15,16}$ Recently, a minor CD8 T cell population of so-called "virtual memory" CD8 T (CD8 $T_{\mathrm{VM}}$ ) cells has been described. ${ }^{17-25}$ These cells arise naturally in naive mice housed under specific pathogen-free and germfree conditions without the exposure to foreign Ag, and display the phenotype and function of Agexperienced "true" memory CD8 T cells. ${ }^{21,22}$ Namely, CD8 T $\mathrm{VM}_{\mathrm{VM}}$ cells rapidly produce IFNy upon TCR stimulation or in response to the cytokines IL-12 and IL-18. They have also been shown to confer
Ag-specific protection against Listeria monocytogenes $(\mathrm{Lm})$ infection. ${ }^{18-20}$ However, whether CD8 $\mathrm{T}_{\mathrm{VM}}$ cells can provide non Agspecific protection is controversial. ${ }^{20,26}$ While Jameson and colleagues conclude that neither virtual nor true memory transgenic CD8 $T$ cells confer non-cognate protection against wild-type $L m$ infection, ${ }^{20} \mathrm{Kedl}$ and colleagues have shown that transgenic CD8 $\mathrm{T}_{\mathrm{VM}}$ cells with irrelevant antigen specificity can mediate bystander protection against $L m$ infection. ${ }^{26}$

It has been shown that IL-4 produced by natural killer T (NKT) cells can drive the generation of CD8 $\mathrm{T}_{\mathrm{VM}}$ cells under steady-state condition in the absence of foreign $\mathrm{Ag}^{23,24}$ This type of CD8 $\mathrm{T}_{\mathrm{VM}}$ cell is abundant in $\mathrm{BALB} / \mathrm{c}$ mice but less so in C57BL/6 mice, ${ }^{23}$ presumably correlating with the relative abundance of IL-4-producing NKT cells in the respective strains. To date, however, it has not been explored whether IL-4 responses generated by helminth infection or immunization regulate the $\mathrm{CD} 8 \mathrm{~T}_{\mathrm{VM}}$ population in a "bystander" fashion, and whether these cells could provide non-cognate innate protection against infections with unrelated pathogens. In this study, we provide fundamentally new insight into the biology of virtual memory CD8 $\mathrm{T}$ cells in the context of IL-4-dominated immune responses to helminth infection or immunization, and reveal their previously unknown protective potential against subsequent unrelated infection.

\section{RESULTS}

Helminth infection confers protection against subsequent bacterial infection

To examine the effects of helminth infection on subsequent bacterial challenge, we infected C57BL/6 (B6) mice with the strictly

\footnotetext{
${ }^{1}$ Trudeau Institute, Saranac Lake, NY 12983, USA

Correspondence: J. S. Lin (jslin@trudeauinstitute.org) or M. Mohrs (markus.mohrs@regeneron.com)

${ }^{2}$ Present address: Regeneron Pharmaceuticals, Inc., Terrytown, NY, USA

${ }^{3}$ Present address: Department of Microbiology and Immunology, University of Texas School of Medicine Health Science Center at San Antonio, San Antonio, TX, USA

These authors contributed equally: K. Mohrs, F. M. Szaba
}

Received: 31 March 2018 Revised: 24 September 2018 Accepted: 30 September 2018

Published online: 25 October 2018 
enteric parasite Heligmosomoides polygurus $(H p)$, drug-cured the animals after 2 weeks, and infected them intraperitoneally with the Gram-positive bacterium Lm 1 week later. In contrast to considerable epidemiological evidence and animal model studies indicating that helminth infection increases susceptibility to viral and bacterial infection, ${ }^{1-5,8}$ we found that mice previously infected with $\mathrm{Hp}$ showed significantly improved survival after challenge with $L m$ compared with their Hp-uninfected counterparts (Fig. 1a). $\mathrm{Hp}$-cured mice also showed significantly reduced bacterial burden in the liver at day 3 after infection (Fig. 1b). Consistent with the survival data, three out of ten naïve controls had succumbed to $\mathrm{Lm}$ infection while all $\mathrm{Hp}$-cured mice survived until analysis at day 3 (Fig. 1b). Even more pronounced protection was observed when $\mathrm{Hp}$-cured mice were challenged orally with the enteric Gramnegative bacterium Yersinia pseudotuberculosis (Yp) (Fig. 1c). $\mathrm{Hp}$ cured mice were significantly protected against $Y p$ infection with nearly $50 \%$ of the mice surviving the lethal $Y p$ challenge with an overall 11-day prolongation in median survival time as compared with naïve controls.

Virtual memory CD8 T cells expand during helminth infection To investigate the possibility that the IL-4-dominated Th2 response to helminth infection drives the generation of CD8 TVM cells, we inoculated B6 IL-4 reporter mice with $\mathrm{Hp}$ and analyzed the draining mesenteric $L N$ (mesLN) 2 weeks later. As expected ${ }^{13}$, the infection elicited a robust CD4 effector $\left(\mathrm{CD} 44^{\text {hi }} \mathrm{CD} 62 \mathrm{~L}^{\mathrm{lo}}\right) \mathrm{T}$ cell response with an abundance of IL-4 (IL-4/GFP ${ }^{+}$) (Fig. 2a). Although CD8 $T$ cells have reportedly no appreciable impact on the immunological or parasitological parameters, ${ }^{14}$ unexpectedly, we detected a substantially increased frequency of central memory (CD44 ${ }^{\text {hi }} \mathrm{CD} 62 \mathrm{~L}^{\text {hi }}$ ) but not effector $\left(\mathrm{CD} 44^{\text {hi }} \mathrm{CD} 62 \mathrm{~L}^{\mathrm{lo}}{ }^{\mathrm{l}}\right.$ ) phenotype CD8 $\mathrm{T}$ cells in $\mathrm{Hp}$-infected mice (Fig. $2 \mathrm{~b}$ ). These $\mathrm{CD} 44^{\text {hi }} \mathrm{CD} 62 \mathrm{~L}{ }^{\text {hi }}$ cells were further identified as CD8 $T_{V M}$ cells based on a CD49d ${ }^{\text {lo }}$ phenotype (Fig. 2b) ${ }^{18,25}$ Over the course of infection, CD8 $T_{V M}$ cells $\left(C D 44^{\text {hi }} C D 49 d^{\text {lo }}\right)$ expanded first in the draining mesLN around day 5 concurrent with the onset of the Th2/IL-4 response (Fig. 2c) $i^{13}$ and then disseminated systemically to non-reactive secondary lymphoid organs, a migration pattern consistent with their $C_{1262}{ }^{h i}$ phenotype. The frequency and number of CD8 $T_{V M}$ cells remained significantly elevated for prolonged periods, even when the mice were drug-cured 2 weeks after infection (Fig. 2d). Moreover, the increase of CD49 $\mathrm{d}^{\mathrm{lo}} \mathrm{CD} 8 \mathrm{~T}_{\mathrm{VM}}$ cells occurred in both B6 mice and BALB/c mice infected with $H p$ (Fig. 2f). Notably, while CD44 was clearly upregulated on activated CD4 T cells in both B6 and BALB/C mice (Fig. 2a, e), CD8 T cells in BALB/c mice failed to display a distinctive shift of CD44 expression (Fig. 2f, upper panels), and CD8 $\mathrm{T}_{\mathrm{VM}}$ cells can only be unambiguously identified by the downregulation of CD49d (Fig. 2f, lower panels). Since
$\mathrm{BALB} / \mathrm{C}$ mice are widely used in helminth disease models, this might explain, at least in part, why the activation of CD8 T cells has largely been unnoticed in that setting. The increased frequency of CD8 $T_{V M}$ cells in BALB/c mice compared with B6 mice correlated with an increased frequency of IL-4/GFP + cells in the mesLN of naive and $\mathrm{Hp}$-infected mice (Fig. $2 \mathrm{~g}$, h). Our data reveal that CD8 $T_{V M}$ cells expanded during helminth infection. The origin of the CD8 $\mathrm{T}_{\mathrm{VM}}$ cell expansion in the IL-4-rich mesLN, ${ }^{13}$ its kinetics, and the comparison of IL-4/Th2-biased BALB/C to B6 mice, together suggest that the abundance of CD8 $\mathrm{T}_{\mathrm{VM}}$ cells is regulated by the production of IL-4 in this model.

The expansion of CD8 $\mathrm{T}_{\mathrm{VM}}$ cell is dependent on direct IL-4 signals To explore whether other IL-4-associated Th2 responses would also result in a CD8 $\mathrm{T}_{\mathrm{VM}}$ cell expansion, we immunized B6 IL-4 reporter mice subcutaneously into the footpad with Schistosoma mansoni eggs or 4-hydroxy-3-nitrophenylacetyl-KLH (NP-KLH) in alum. Both agents induced robust Th2/IL-4 responses in the draining popliteal LN and resulted in a significantly increased frequency of CD8 $\mathrm{T}_{\mathrm{VM}}$ cells (Fig. 3a). Of note, the extent of the CD8 $\mathrm{T}_{\mathrm{VM}}$ cell expansion correlated with the frequency of $\mathrm{IL}-4^{+}$cells, further supporting the hypothesis that IL-4 governs the expansion of CD8 $T_{\mathrm{VM}}$ cells. To explore this further, we activated glycolipidspecific invariant NKT (iNKT) cells, potent producers of IL- $4,{ }^{27}$ by intravenous administration of the glycolipid, non-protein antigen aGalactosylceramide (aGalCer). The administration of aGalCer to B6 wild-type (WT) mice but not iNKT-deficient CD1d KO mice resulted in an increased frequency (Fig. $3 \mathrm{~b}$ ) and number (data not shown) of CD8 $\mathrm{T}_{\mathrm{VM}}$ cells. Notably, even under steady-state conditions $\mathrm{CD} 8 \mathrm{~T}_{\mathrm{VM}}$ cells were significantly reduced in naive CD1d KO mice (Fig. 3b), corroborating previous studies that iNKT cells contribute to the regulation of the CD8 $T_{V M}$ abundance, potentially by production of IL-4. ${ }^{23,24}$ As expected, CD8 T $T_{V M}$ cells were also significantly reduced in naive and aGalCer-immunized IL-4 KO B6 mice (Fig. 3c), demonstrating a critical role for IL-4 in driving the expansion of CD8 $\mathrm{T}_{\mathrm{VM}}$ pool. Interestingly, CD8 $\mathrm{T}_{\mathrm{VM}}$ cells expanded in IL-4 KO B6 mice upon aGalCer immunization to some degree, revealing a partially IL-4-independent mechanism. Together, our data suggests that IL- 4 production elicited by diverse immune stimuli in various sites results in the expansion of the CD8 $\mathrm{T}_{\mathrm{VM}}$ pool.

To revisit the role of IL-4 in the expansion of CD8 $T_{V M}$ cells during natural infection, we challenged WT and IL-4 KO mice on $\mathrm{B} 6$ and $\mathrm{BALB} / \mathrm{c}$ backgrounds with $\mathrm{Hp}$. CD8 $\mathrm{T}_{\mathrm{VM}}$ cells were significantly reduced in naive and $\mathrm{Hp}$-infected IL-4 KO mice on both genetic backgrounds (Fig. 3d). Interestingly, while CD8 $\mathrm{T}_{\mathrm{VM}}$ cells in IL-4 KO mice on the B6 background showed a partial expansion upon $\mathrm{Hp}$ infection, they did not expand in IL-4 KO mice
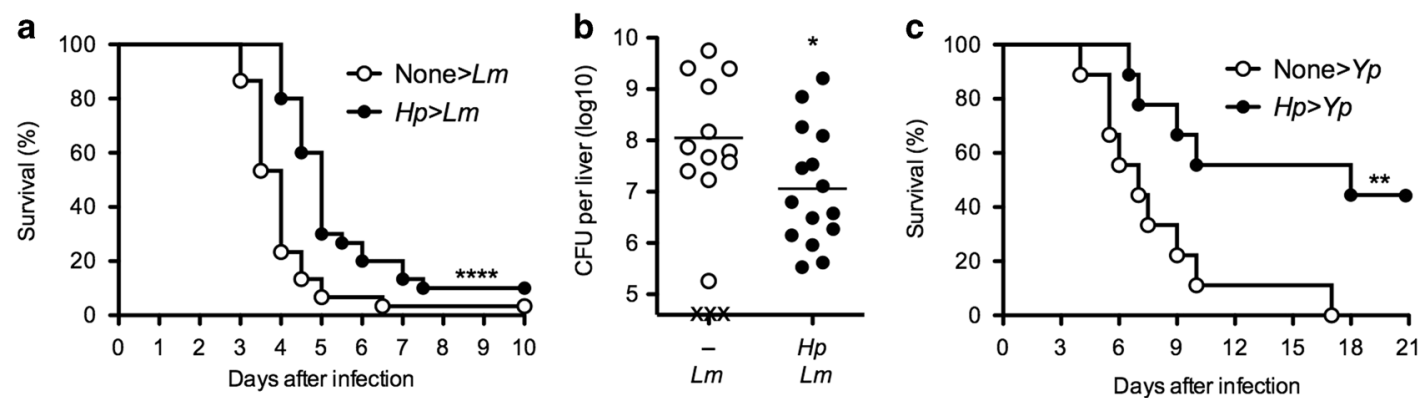

Fig. 1 Helminth infection provides protection against subsequent systemic and enteric bacterial infection. B6 WT mice were either uninfected or infected by gavage with 200 larvae of $\mathrm{Hp}$, and cured with an antihelminthic after 2 weeks. a, b One week later, mice were challenged intraperitoneally with $2.5 \times 10^{6} \mathrm{CFU}$ of the Gram-positive bacterium $\mathrm{Lm}$. a Survival was monitored in $12 \mathrm{~h}$ intervals. Data were pooled from three independent experiments $(n=30$ per group). b Bacterial burden in the liver was determined at day 3 after $L m$ challenge. Data were pooled from two independent experiments. Solid bar depicts mean. $X$ indicates individual mice that had succumbed to infection prior to analysis. c One week after drug cure, mice were challenged by gavage with $5 \times 10^{9} \mathrm{CFU}$ of the Gram-negative bacterium Yp. Survival was monitored in $12 \mathrm{~h}$ intervals. ( $n=9$ per group). ${ }^{*} P<0.05 ;{ }^{* *} P<0.01 ;{ }^{* * *} P<0.0001$ by log rank test $(\mathbf{a}, \mathbf{b})$ or Student's $t$ test $(\mathbf{b})$ 
a
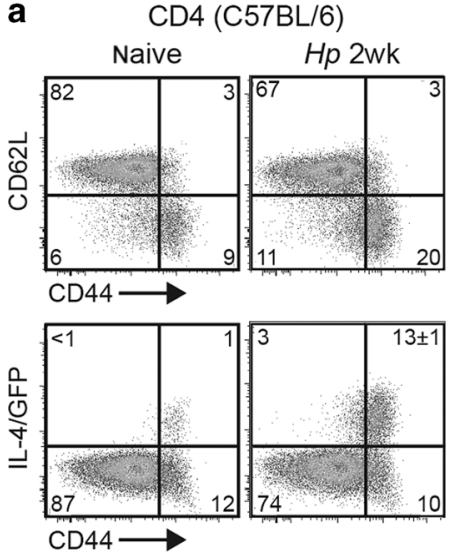

e
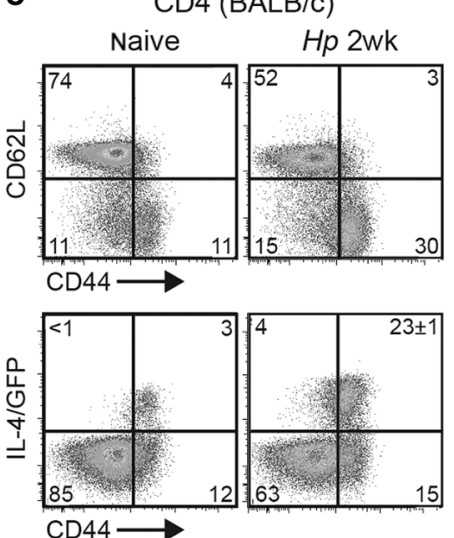

b

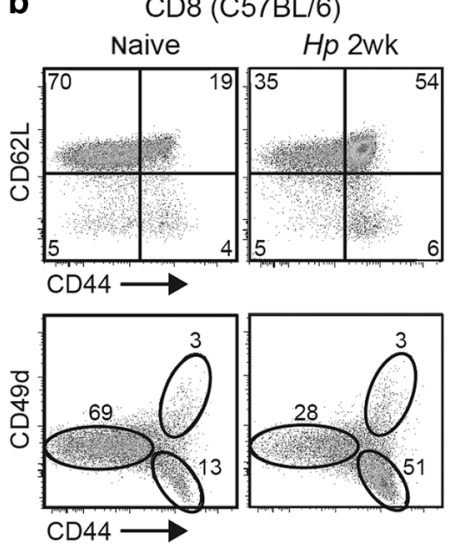

f
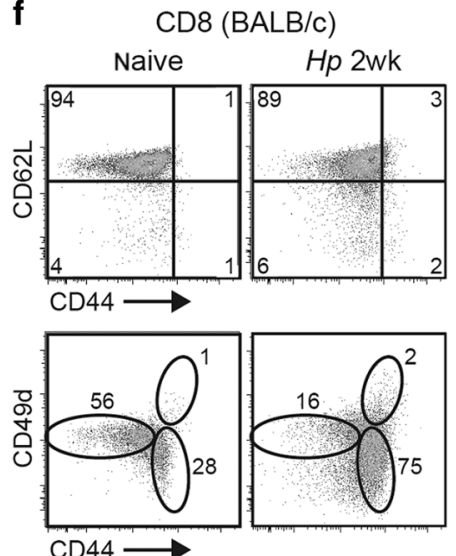

c

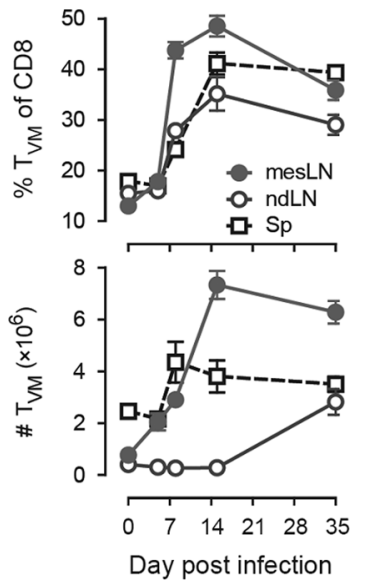

g

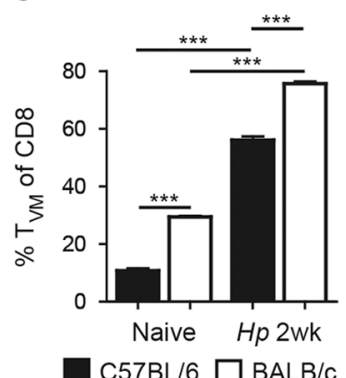

C57BL/6 $\square \mathrm{BALB} / \mathrm{c}$ d
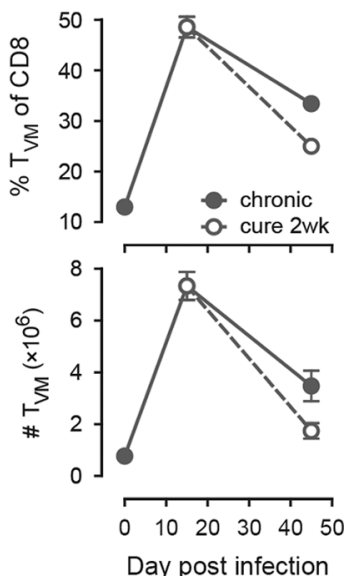

h

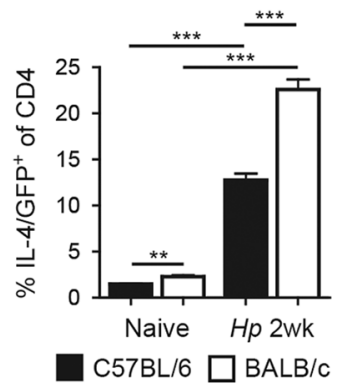

Fig. 2 CD8 $\mathrm{T}_{\mathrm{VM}}$ cells expand upon helminth infection. B6 (a-d, $\left.\mathbf{g}, \mathbf{h}\right)$ or BALB/c (e-h) IL-4 reporter mice were infected with Hp, and the draining mesLN, non-draining LN (ndLN) and spleen (Sp) were harvested at the indicated times and cells were analyzed by FACS. Plots and graphs were gated on $\mathrm{CD}^{+}(\mathbf{a}, \mathbf{e}, \mathbf{h})$ or $\mathrm{CD} 8 \alpha^{+}(\mathbf{b}-\mathbf{d}, \mathbf{f}, \mathbf{g})$ cells. Data in $(\mathbf{d})$ depict the mesLN. Data are representative of two or more independent experiments with three to five mice per group. Error bars depict the SEM. ${ }^{* *} P<0.001$ by Student's $t$ test

on the BALB/c background. Similar outcomes were also observed in IL-4 receptor a chain (IL-4Ra) KO and signal transducer and activator of transcription (STAT) $6 \mathrm{KO}$ mice on the BALB/C background (data not shown). This suggests that the expansion of CD8 $T_{V M}$ cells in BALB/C mice is strictly dependent on IL-4 and IL-4Ra, whereas both IL-4- or IL-4Ra-deficiency can partially be compensated by alternative pathways in $\mathrm{B} 6$ mice infected with $\mathrm{Hp}$ or immunized with aGalCer.

To establish whether the CD8 $\mathrm{T}_{\mathrm{VM}}$ population is regulated by direct IL-4Ra signals, we generated $\mathrm{B} 6$ and $\mathrm{BALB} / \mathrm{C}$ radiation chimeras reconstituted with equal parts of genetically marked WT and IL-4Ra KO bone marrow (BM) cells. As shown in Fig. 3e, the frequency of CD8 $T_{V M}$ cells was significantly reduced in IL-4Ra KO CD8 $\mathrm{T}$ cells in both naïve and $\mathrm{Hp}$-infected chimeras on both genetic backgrounds. Consistent with the IL-4 KO data (Fig. 3d), IL-4Ra KO cells in B6 mice showed a partial expansion of CD8 $T_{V M}$ cells upon $\mathrm{Hp}$ infection but remained significantly reduced compared to their WT counterparts in the same animal, whereas IL-4Ra KO CD8 TVM cells in BALB/C mice did not expand at all (Fig. 3e). Together, these data reveal that direct IL-4Ra signals regulate the size of CD8 $T_{V M}$ pool in naive animals and upon IL-4-dominated Th2 responses to immunization or infection. However, the extent to which IL-4 and IL$4 \mathrm{Ra}$ signals govern the expansion depends on the genetic background. While the expansion of CD8 $T_{V M}$ cell in BALB/C mice is strictly dependent on IL-4 and IL-4Ra, alternative pathways can partially compensate for IL-4 and IL-4Ra in B6 mice.
The expansion of CD8 $T_{V M}$ cell is independent of cognate antigen The robust expansion of CD8 $\mathrm{T}_{\mathrm{VM}}$ cells without apparent effector cell differentiation (Fig. 2b, $f$ ) suggests that CD8 $T_{V M}$ cells do not encounter cognate antigen during this process. Moreover, CD8 $\mathrm{T}_{\mathrm{VM}}$ cells also expanded without the administration of exogenous protein antigen when iNKT cells were selectively activated by aGalCer (Fig. 3b, c). To follow a population of CD8 T cells of defined, infection-irrelevant specificity, we transfer OT-I TCR transgenic CD8 T cells specific for $\mathrm{OVA}_{257-264}$ into CD45.1 ${ }^{+}$ congenic B6 WT mice, and infected the recipients with $\mathrm{Hp}$ the following day. Like in the polyclonal host CD8 $\mathrm{T}_{\mathrm{VM}}$ cell population, $\mathrm{Hp}$ infection resulted in a marked increase in the frequency and number of CD8 $T_{V M}$ cells in the OT-I population as well (Fig. 4). This indicates that the expansion of CD8 $\mathrm{T}_{\mathrm{VM}}$ cells during $\mathrm{Hp}$ infection is independent of cognate antigen. Collectively, our data support a model whereby the expansion of CD8 $\mathrm{T}_{\mathrm{VM}}$ cells upon IL-4dominant immune responses to infection or immunization occurs independent of the encounter with cognate antigen and is driven predominantly by cytokine.

CD8 $T_{V M}$ cells are sufficient to confer innate non-cognate protection against bacterial infection

CD8 $\mathrm{T}_{\mathrm{VM}}$ cells share many functional features with antigenexperienced "true memory" CD8 T cells, including rapid IFNY production upon TCR stimulation and confer Ag-specific protection against infection. ${ }^{18-20}$ Moreover, they both produce IFNY in 
a

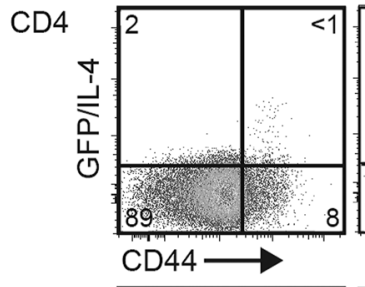

CD8

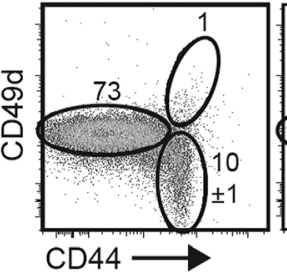

d

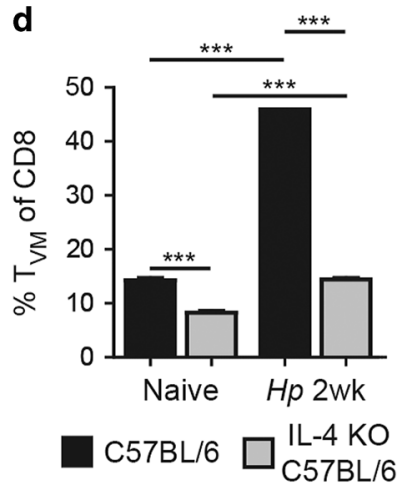

S. mansoni eggs NP-KLH/alum
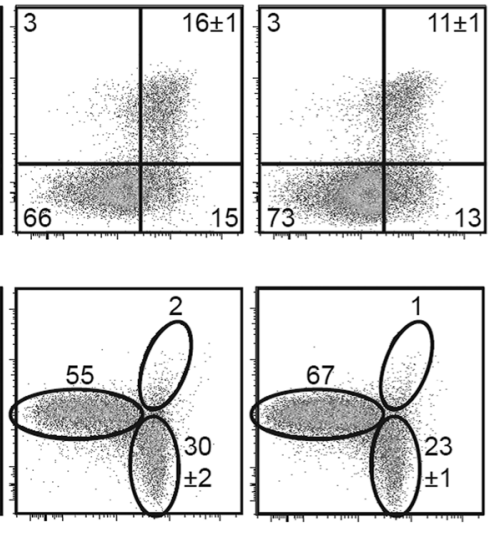

b

C
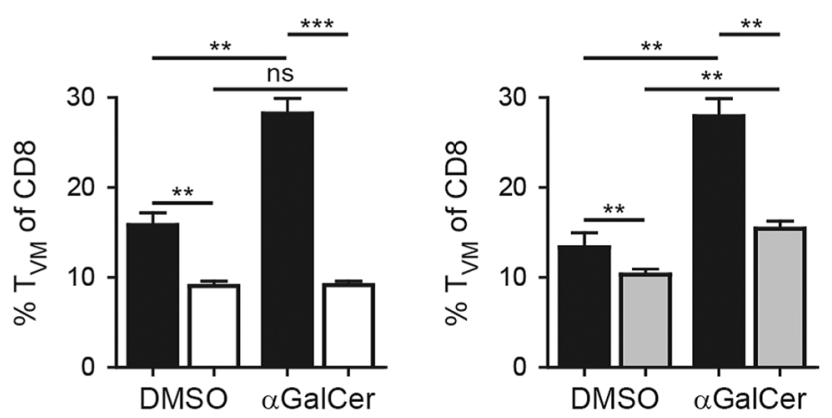

C57BL/6 $\square$ CD1d KO

C57BL/6 $\square$ IL-4 KO

e

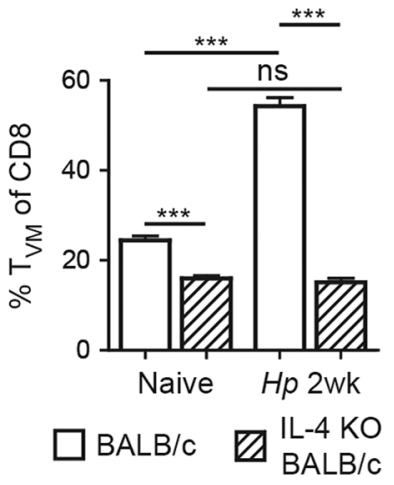

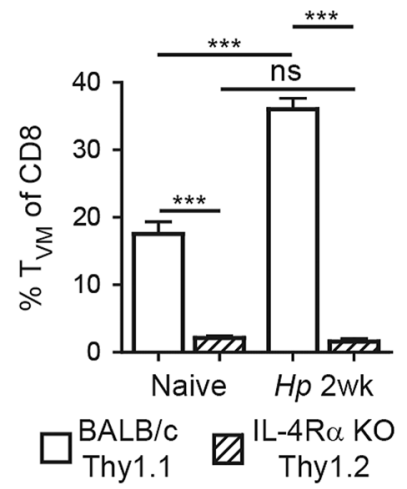

Fig. 3 The expansion of CD8 $\mathrm{T}_{\mathrm{VM}}$ cells is dependent on direct IL-4 signal. a B6 IL-4 reporter mice were immunized with 2500 S. mansoni eggs or $100 \mu \mathrm{g} \mathrm{NP}-\mathrm{KLH} /$ alum by injection into the footpad. The draining popliteal LNs were harvested 9 days later and analyzed as described in Fig. 2 (b, c) B6 WT, CD1d KO (b) or IL-4 KO (c) mice were immunized intravenously with $0.5 \mu \mathrm{g} \alpha \mathrm{GalCer}$. Control mice were treated with solvent alone (PBS containing BSA and DMSO). The spleen was harvested 1 week later and CD8 $\alpha^{+}$T cells were analyzed as described in Fig. 2. d WT or IL-4 $\mathrm{KO}$ mice in $\mathrm{B} 6$ or $\mathrm{BALB} / \mathrm{c}$ background either remained uninfected or were infected with $\mathrm{Hp}$. The mesLN cells were harvested 2 weeks later and analyzed as described in Fig. 2. e Mixed BM chimeras were generated by reconstituting equal part of irradiated CD45.1 ${ }^{+}$B6 recipients with WT $\left(\mathrm{CD} 45.1^{+}\right)$and IL-4R $\alpha \mathrm{KO}\left(\mathrm{CD} 45.2^{+}\right) \mathrm{BM}$, or Thy $1.1^{+}$BALB/C recipients with WT (Thy $\left.1.1^{+}\right)$and IL-4R $\alpha \mathrm{KO}\left(\right.$ Thy $\left.1.2^{+}\right)$BM. Reconstituted mice either remained uninfected or were infected with $\mathrm{Hp}$. The mesLN cells were harvested 2 weeks later, and WT and IL-4R $\alpha$ KO CD8 T cells were analyzed by gating on the respective congenic marker. Data are representative of at least two independent experiments with three to five mice per group. Error bars depict the SEM. ns, not significant; ${ }^{* *} P<0.01 ;{ }^{* * *} P<0.001$ by Student's $t$ test
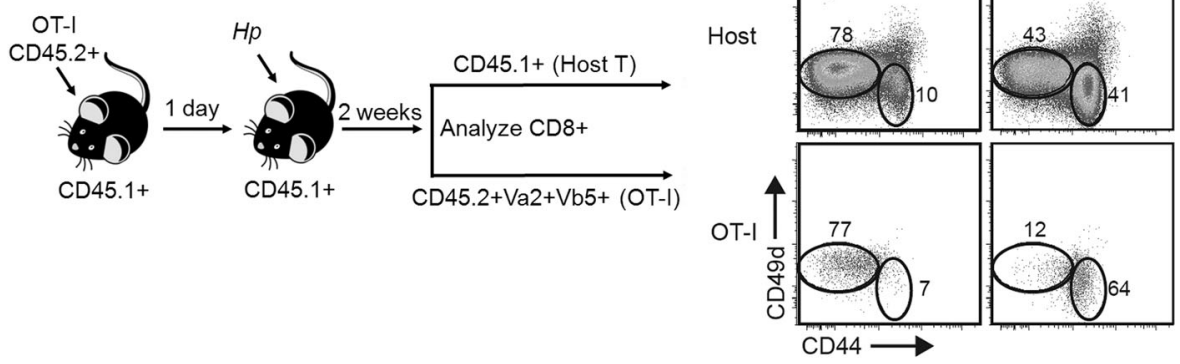

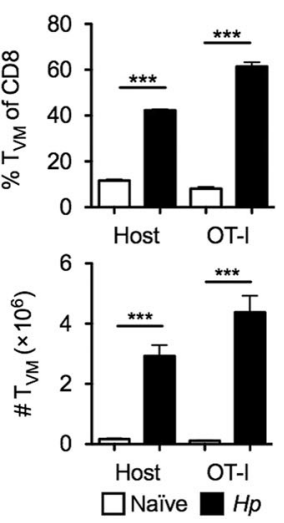

Fig. 4 The expansion of $C D 8 T_{V M}$ cell after helminth infection is independent of cognate antigen. OT-I TCR transgenic cells were transferred intravenously into CD45.1 ${ }^{+}$congenic B6 mice. One day later the recipient mice either remained uninfected or were infected with Hp. Two weeks later, host T cells (CD8 $\left.\alpha^{+} \mathrm{CD} 45.1^{+}\right)$and OT-I cells $\left(\mathrm{CD} 8 \alpha^{+} \mathrm{CD} 45.2^{+} \mathrm{V} \alpha 2^{+} \mathrm{V} \beta 5^{+}\right)$in the mesLN were analyzed as described in Fig. 2. Data are representative of two independent experiments with three to four mice per group. Error bars depict the SEM. ${ }^{* * *} P<0.001$ by Student's $t$ test 
response to the innate cytokines $\mathrm{IL}-12$ and $\mathrm{IL}-18 .{ }^{18}$ It has been shown that Ag-experienced memory CD8 $\mathrm{T}$ cells can provide IFNYdependent protection against $L m$ infection in the absence of cognate Ag. ${ }^{28}$ Along with the observations that helminth infection provided increased resistance against bacterial challenge (Fig. 1) and induced robust expansion of $\mathrm{CD} 8 \mathrm{~T}_{\mathrm{VM}}$ cells (Fig. 2), we consider the possibility that CD8 $\mathrm{T}_{\mathrm{VM}}$ cells expanded during helminth infection contribute to the non-cognate protection against unrelated bacteria. Since $H p$ infection elicits extensive immunological and physiological changes in the parasitized host, in order to definitively demonstrate that CD8 $T_{V M}$ cells directly contribute to the increased protection, and to explore whether CD8 $T_{V M}$ cells alone are sufficient to confer protection, we sorted naïve $\left(C D 44^{\text {lo }}\right)$ and CD8 $T_{V M}\left(C D 44^{\text {hi }} C D 49 d^{\text {lo }}\right)$ cells from the same $\mathrm{Hp}$-infected donors and transferred them separately into naïve recipients (Fig. 5). To avoid the possibility that IFNy produced by host cells masks the potential protective impact of the transferred CD8 T cells, we used IFNY KO recipients. ${ }^{28}$ As shown in Fig. 5, transfer of CD8 $T_{\mathrm{VM}}$ cells but not naïve CD8 T cells from the same donor reduced the bacterial burden at least 10-fold in both liver and spleen, demonstrating that CD8 $\mathrm{T}_{\mathrm{VM}}$ cells expanded during helminth infection not only directly contribute to but also suffice to confer innate non-cognate protection against bacterial challenge.

\section{DISCUSSION}

While the signature Th2 cytokine IL-4 has previously been linked to the expansion of memory phenotype CD8 T cells ${ }^{15,29,30}$ and the size of the CD8 $T_{V M}$ pool under steady-state conditions, ${ }^{23,24,31}$ to our knowledge, we are the first to describe that Th2 responses to helminth infection or immunization result in the antigenindependent systemic "bystander" expansion of CD8 TVM cells in both $\mathrm{B} 6$ and BALB/C mice. The expansion of CD8 $T_{V M}$ cells was strictly dependent on IL-4 and direct IL-4Ra signals in BALB/C mice; interestingly, it was partially IL-4 and IL-4Ra-independent in B6 mice (Fig. 3c, e). Since the IL-4Ra chain is indispensable for IL-13 signaling mediated exclusively by the type II IL-4 receptor complex, IL-13 cannot compensate for IL-4 in IL-4Ra-deficient B6 mice. ${ }^{32-34}$ This conclusion is consist with the general understanding that $\mathrm{T}$ cells do not express the IL-13Ra1 chain and do not respond to IL-13, 32,34 although it has been shown that Th17 polarized CD4 + T cells, but no other T cell subsets, can express a functional IL-13 receptor. ${ }^{35}$ Therefore, our data suggest that the expansion of CD8 $T_{V M}$ cell after helminth infection is dominantly dependent on IL-4. In support of our conclusion, it has been reported that the administration of IL-4/anti-IL-4 antibody complexes into naïve B6 mice as well as OT-I TCR transgenic mice could induce the generation of memory-like CD8 T cell in the periphery. ${ }^{36}$ It is conceivable that IL-15-which is also critical for CD8 $\mathrm{T}_{\mathrm{VM}}$ cell development and can induce the expansion of CD8 $\mathrm{T}_{\mathrm{VM}}$ cells in vivo ${ }^{25,26,37}$ - could partially compensate the absence of IL-4 or IL-4Ra, particularly when diverse cellular subsets are engaged during complex immune responses to infections (Fig. 2d, e). However, it is less clear how this occurs upon the selective activation of iNKT cells by aGalCer (Fig. 2c). Nevertheless, how alternative pathway(s) are activated in the diverse settings of Th2 immunity and why it is limited to the B6 background warrants further investigation.

More intriguingly, our data presented here provide direct evidence that CD8 $\mathrm{T}_{\mathrm{VM}}$ cells expanded during helminth infection are in turn sufficient to increase resistance to bacterial challenge, suggesting that they can confer enhanced non-cognate protection in helminth-infected mice. This unexpected CD8 $\mathrm{T}_{\mathrm{VM}^{-}}$ mediated non-cognate protection is broadly effective against infection with both Gram-negative and Gram-positive bacteria via different routes. Notably, the protection conferred by CD8 $\mathrm{T}_{\mathrm{VM}}$ cells is not as potent as that provided by antigen-specific virtual or true memory T cells. ${ }^{19,20}$ However, considering its innate-like non Ag-specific feature, we speculate that the IL-4-mediated expansion of CD8 $T_{V M}$ cells during helminth infection has evolved to provide broad "collateral protection" against diverse secondary infections in an Ag-independent manner. Furthermore, our data show that IL-4-inducing adjuvants, such as the routinely used
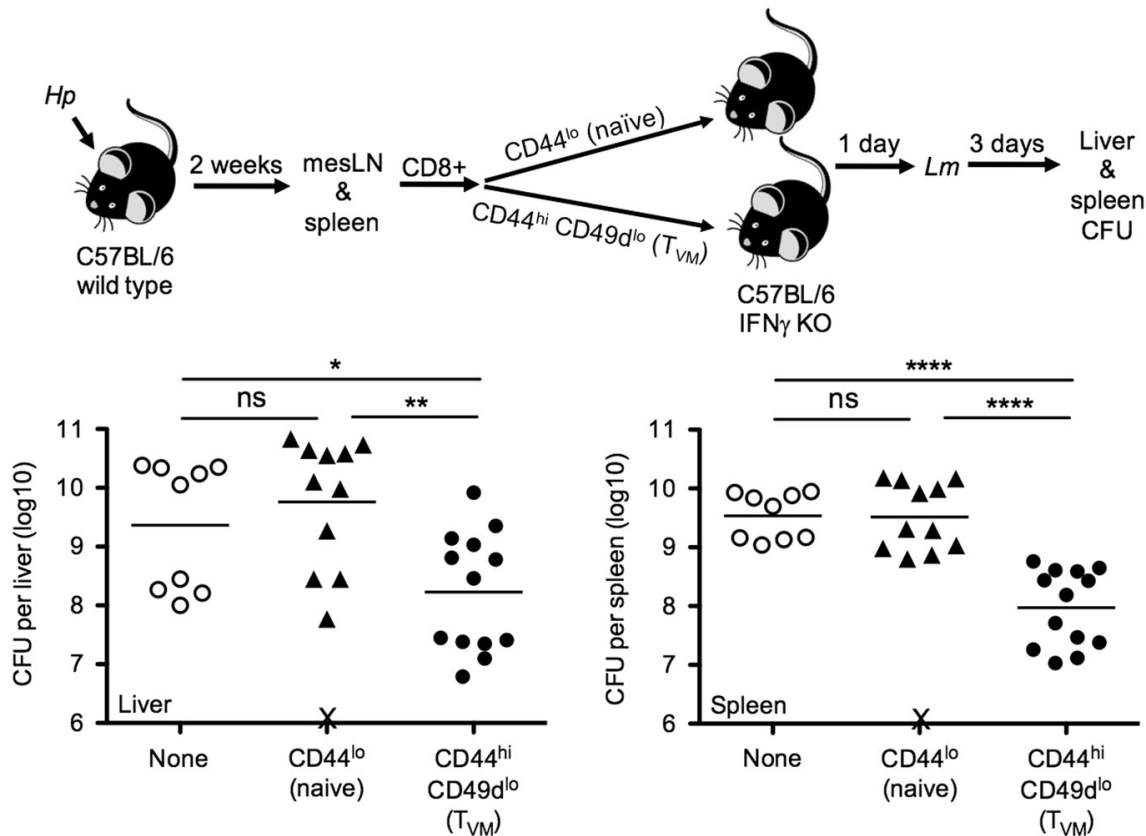

$\left(\mathrm{T}_{\mathrm{VM}}\right)$

Fig. $5 \mathrm{CD} 8 \mathrm{~T}_{\mathrm{VM}}$ cells are sufficient to confer innate non-cognate protection against bacterial infection. B6 WT mice were infected with $\mathrm{Hp}$ and naïve $\left(C D 44^{\mathrm{lo}}\right)$ or $\mathrm{CD} 8 \mathrm{~T}_{\mathrm{VM}}\left(\mathrm{CD} 44^{\mathrm{hi}} \mathrm{CD} 49 \mathrm{~d}^{\mathrm{lo}}\right)$ cells were sorted from the pooled mesLN and spleen 2 weeks later. A total of $2 \times 10^{6}$ cells were transferred into naïve IFN $\gamma$ KO recipients, which were infected with $2 \times 10^{5} \mathrm{Lm}$ the next day. Bacterial burden in liver and spleen was determined at day 3. Data were pooled from two independent experiments. Solid bar depicts mean. $\mathrm{X}$ indicates individual mice that had succumbed to infection prior to analysis. ns, not significant; ${ }^{*} P<0.05$; ${ }^{* *} P<0.01 ;{ }^{* * *} P<0.0001$ by one-way ANOVA 
alum, one of the only two FDA-approved adjuvants, increase the abundance of CD8 $\mathrm{T}_{\mathrm{VM}}$ cells and subsequently have a profound impact on the systemic CD8 T cell population. It stands to reason that IL-4-associated asthmatic and allergic disorders may have a similar effect on CD8 $T_{V M}$ cells and the systemic CD8 T cell pool.

It is worth noting that in published studies the protective potential of CD8 $T_{V M}$ cells was typically tested in model infections such as $L m$ and lymphocytic choriomeningitis virus (LCMV) in relatively clean systems that only involved CD8 $\mathrm{T}_{\mathrm{VM}}$ cells. ${ }^{19,20,26,37,38}$ The protection was suggested to be mediated by the production of IFNy by CD8 $\mathrm{T}_{\mathrm{VM}}$ cells. ${ }^{18}$ In contrast, helminth infection elicits complex immune responses, and the impact on the subsequent infection was tested in various infection models with various combinations of pathogens. Therefore, the reported outcomes were highly diverse and likely highly context dependent. While most studies conclude that helminthiases negatively impact immunity to concurrent and/or subsequent bacterial or viral infection, ${ }^{1-7}$ there are also reports of either positive effects $^{39,40}$ or no impact. ${ }^{7,41,42}$ The mechanism(s) underlying this discrepancy remain poorly understood, and could be specific for particular infection models, different combinations of pathogens, different timing or route of infection, or a number of other factors. Consequently, care must be taken when interpreting and comparing these results. Nonetheless, our data demonstrate that prior $\mathrm{Hp}$ infection can increase host resistance to subsequent bacterial infections. To the best of our knowledge, we are the first to suggest that helminth-mediated effects could provide survival advantage to subsequent unrelated infections through the expansion of CD8 T a transfer model that CD8 $\mathrm{T}_{\mathrm{VM}}$ cells expanded upon helminth infection suffice to reduce bacterial burden, we did not rule out the possibility that other factors may also contribute to the enhanced protection in $\mathrm{Hp}$-infected mice. Moreover, the defense mechanisms that control subsequent infections may vary between pathogens. Thus, in some infectious contexts, the protective potential of CD8 $\mathrm{T}_{\mathrm{VM}}$ cells may not be relevant or may be counteracted by other profound mechanisms. Potential mechanisms by which bystander infection may affect immune responses to unrelated pathogens have been discussed. ${ }^{1,43}$ Further studies will be required to investigate the difference between beneficial and detrimental effects, as well as to determine other potential factors enhancing protection observed in our model.

Recently, awareness has grown regarding the shortcomings of mouse models in studying immune responses to infections. ${ }^{43,44}$ While laboratory mice are deliberately housed under specific pathogen-free conditions, humans acquire a diverse and individual history of acute and chronic infections throughout life. The interplay between different pathogens and the immune responses they elicit are complex. Consequently, while the infection history of an individual is a likely determinant of immune responses, it is also a potential confounding factor in studying and understanding immune responses to subsequent infection. The sequential infection of mice with multiple pathogens may allow modeling these events to study the complex interactions that can translate to humans. ${ }^{43,44}$ Indeed, broad "collateral protection" resulting from an individual's immunological history might be widespread, particularly in helminth endemic areas where exposure to secondary infection is common, and in populations where children received alum-based vaccines. Harnessing the protective reality of virtual memory CD8 T cells might open new avenues for prophylactic and therapeutic intervention.

\section{METHODS}

Mice

Wild-type mice were on both the C57BL/6 and the BALB/C background, as were IL-4 reporter mice, B6.129- $/ / 4^{\text {tm } 1 L k y} / \mathrm{J}$ and C.129- $/ / 4^{\text {tm } 1 L k y} / \mathrm{J}$, respectively. ${ }^{45,46} \mathrm{IL}-4 \mathrm{KO}$ and IL-4Ra KO $\mathrm{KO}^{33}$ mice were also on both the $\mathrm{C} 57 \mathrm{BL} / 6$ and the BALB/C background. $\mathrm{CD} 1 \mathrm{~d}$ KO (B6(C)-Cd1d1/Cd1d2 $\left.2^{\text {tm1.2Aben }} / \mathrm{J}\right)$, IFNץ KO (B6.129S7-Ifng $4^{\text {tm1Ts }}$ ), and OT-I TCR transgenic mice specific for OVA $257-264$ in the context of $\mathrm{H}_{2} \mathrm{~K}^{\mathrm{b}}$ (C57BL/6-Tg(TcraTcrb) $\left.1100 \mathrm{Mjb} / \mathrm{J}\right)$ were on the C57BL/6 background. CD45.1 congenic mice (B6.SJL-Ptprc ${ }^{a}$ Pepc $^{b} /$ BoyJ) were on the C57BL/6 background, and Thy 1.1 congenic mice (CBy.PL (B6)-Thy $1^{a} / \mathrm{ScrJ}$ ) were on the BALB/c background. Animals were bred and kept under specific pathogen-free conditions at the Trudeau Institute and were used at 8-12 weeks of age. All experiments were performed under Institutional Animal Care and Use Committee-approved protocols at the Trudeau Institute.

\section{Infections and immunizations}

Animals were infected by gavage with 200 third-stage larvae of $\mathrm{Hp}$ as previously described. ${ }^{45}$ Where indicated, mice were treated by gavage with the antihelminthic pyrantel pamoate $(100 \mathrm{mg} / \mathrm{kg})$ 2 weeks later to terminate $\mathrm{Hp}$ infection. One week later, mice were infected intraperitoneally with $2.5 \times 10^{6} \mathrm{CFU}$ of $\mathrm{Lm}$ (strain EGD) or $5 \times 10^{9}$ CFU of $Y p$ (serotype 0:1 strain 32777) by gavage as previously described. ${ }^{47,48}$ In some experiments, mice were injected with 2500 S. mansoni eggs (Puerto Rican strain NMRI) subcutaneously into the footpad. In other experiments, mice were immunized subcutaneously into the footpad with $100 \mu \mathrm{g}$ of 4hydroxy-3-nitrophenylacetyl-KLH (NP-KLH; Biosearch Technologies) precipitated in alum (Imject Alum; ThermoScientific), or intravenously with $0.5 \mu \mathrm{g} / \mathrm{mouse}$ aGalactosylceramide (aGalCer) in PBS containing $0.1 \%$ BSA and $<0.25 \%$ DMSO, or solvent alone (PBS containing BSA and DMSO). Mice were sacrificed and cells were harvested and analyzed at the indicated times. For survival studies, mice were monitored in $12 \mathrm{~h}$ intervals. Unresponsive or recumbent mice were considered moribund and euthanized. Bacterial burden in liver and spleen was determined at day 3 after Lm infection as previously described. ${ }^{47}$

\section{Flow cytometry}

Single cell suspensions were prepared from the mesenteric $L N$, non-draining LN (pooled inguinal, brachial and axillary), and spleen; then stained, acquired on a FACS Canto II (BD Biosciences) and analyzed using FlowJo software (Tree Star, Inc.) as described. ${ }^{45}$ Dead cells were excluded from the analyses by the addition of propidium iodide $(0.5 \mu \mathrm{g} / \mathrm{ml}$; Sigma-Aldrich). The following mAbs were used and clone designations are given in parenthesis: CD4 (RM4-5), CD8a (53-6.7), CD44 (IM7), CD45.1 (A20), CD45.2 (104), CD49d (R1-2 or 9C10), CD62L (MEL-14), IL-4Ra (M1), Thy1.1 (HIS5), Thy1.2 (53-2.1), Va2 (B20.1), Vß5 (MR9-4).

\section{Mixed bone marrow chimeras}

Bone marrow cells from WT and IL-4Ra KO mice on either the B6 or $\mathrm{BALB} / \mathrm{c}$ background were mixed at a 1:1 ratio, and a total of $1 \times$ $10^{7}$ cells were injected intravenously into lethally irradiated (950 rad provided in two doses) WT recipients on the same background. Chimeric mice were allowed to immune-reconstitute for 6-8 week before they were infected with $\mathrm{Hp}$.

\section{CD8 $\mathrm{T}$ cell isolation and transfer}

$\mathrm{CD}^{+}$cells were enriched from the spleens and LN of OT-I TCR transgenic mice by $B$ cell panning and $1 \times 10^{7}$ cells were transferred intravenously into CD45.1 congenic hosts one day prior to $\mathrm{Hp}$ infection. To sort naïve $\left(\mathrm{CD} 44^{\mathrm{lo}}\right)$ and $\mathrm{CD} 8 \mathrm{~T}_{\mathrm{VM}}$ $\left(\mathrm{CD} 44^{\mathrm{hi}} \mathrm{CD} 49 \mathrm{~d}^{\mathrm{lo}}\right)$ cells, B6 mice were infected with $\mathrm{Hp}$ and CD8 $T$ cells from the mesLN and spleen were enriched by negative selection using magnetic activated cell sorting (MACS; Miltenyi Biotec Inc) according to the manufacturer's instruction. CD8enriched samples were then stained for CD8a, CD44, and CD49d and the designated populations were sorted on an InFlux cell sorter (BD Biosciences). A total of 1-3 $\times 10^{6}$ cells of the respective populations were transferred intravenously into IFN $\gamma \mathrm{KO}$ recipients one day prior to $L m$ infection. 


\section{Statistical analysis}

Prism 5 (GraphPad Software) was used for statistical analysis. Data sets were compared by unpaired, two-tailed Student's $t$ test or one-way ANOVA. Data are represented as mean \pm SEM if not indicated otherwise. Survival data were analyzed by the log rank test. ns, not significant; ${ }^{*} P<0.05 ;{ }^{* *} P<0.01 ;{ }^{* *} P<0.001$; $* * * * P<0.0001$.

\section{ACKNOWLEDGEMENTS}

We thank Dr. Edward Pearce (Max Planck Institute of Immunology and Epigenetics, Germany) for providing S. mansoni eggs; Dr. James B. Bliska (Geisel School of Medicine at Dartmouth) for providing Y. pseudotuberculosis; Dr. Marcia Blackman for helpful discussion of this project; Dr. Lawrence Johnson for critical reading of the manuscript and assistance with statistical analyses; Debra Duso for technical assistance; and the dedicated staff of the Trudeau Institute Animal Facility for the expert breeding and care of mice. This work was supported by funds from Trudeau Institute (IHP-886 to JSL) and the National Institutes of Health grants Al061577 (JSL), Al104788 (EAL) and Al076479 (MM).

\section{AUTHOR CONTRIBUTIONS}

J.S.L. and M.M. designed the studies, analyzed the data and wrote the manuscript. J.S. L., K.M., F.M.S., and L.W.K. performed experiments and analyzed the data. E.A.L. contributed aGalCer and CD1d KO mice.

\section{ADDITIONAL INFORMATION}

Competing interests: The authors declare no competing interests.

\section{REFERENCES}

1. Stelekati, E. \& Wherry, E. J. Chronic bystander infections and immunity to unrelated antigens. Cell. Host. Microbe 12, 458-469 (2012).

2. Reese, T. A. et al. Helminth infection reactivates latent gamma-herpesvirus via cytokine competition at a viral promoter. Science 345, 573-577 (2014).

3. Osborne, L. C. et al. Virus-helminth coinfection reveals a microbiota-independent mechanism of immunomodulation. Science 345, 578-582 (2014).

4. Potian, J. A. et al. Preexisting helminth infection induces inhibition of innate pulmonary anti-tuberculosis defense by engaging the IL-4 receptor pathway. J. Exp. Med. 208, 1863-1874 (2011)

5. Monin, L. et al. Helminth-induced arginase-1 exacerbates lung inflammation and disease severity in tuberculosis. J. Clin. Invest. 125, 4699-4713 (2015).

6. Su, C. et al. Helminth-induced alterations of the gut microbiota exacerbate bacterial colitis. Mucosal Immunol. 11, 144-157 (2018).

7. Apiwattanakul, N., Thomas, P. G., Kuhn, R. E., Herbert, D. R. \& McCullers, J. A. Helminth infections predispose mice to pneumococcal pneumonia but not to other pneumonic pathogens. Med. Microbiol. Immunol. 203, 357-364 (2014).

8. Salgame, P., Yap, G. S. \& Gause, W. C. Effect of helminth-induced immunity on infections with microbial pathogens. Nat. Immunol. 14, 1118-1126 (2013).

9. Reynolds, L. A., Finlay, B. B. \& Maizels, R. M. Cohabitation in the intestine: interactions among helminth parasites, bacterial microbiota, and host immunity. J. Immunol. 195, 4059-4066 (2015)

10. Mishra, P. K., Palma, M., Bleich, D., Loke, P. \& Gause, W. C. Systemic impact of intestinal helminth infections. Mucosal Immunol. 7, 753-762 (2014).

11. Anthony, R. M. et al. Protective immune mechanisms in helminth infection. Nat. Rev. Immunol. 7, 975-987 (2007).

12. Maizels, R. M. \& Yazdanbakhsh, M. Immune regulation by helminth parasites: cellular and molecular mechanisms. Nat. Rev. Immunol. 3, 733-744 (2003).

13. Perona-Wright, G., Mohrs, K. \& Mohrs, M. Sustained signaling by canonical helper $T$ cell cytokines throughout the reactive lymph node. Nat. Immunol. 11, 520-526 (2010).

14. Urban, J. F. Jr., Katona, I. M. \& Finkelman, F. D. Heligmosomoides polygyrus: CD4 +but not $\mathrm{CD} 8+\mathrm{T}$ cells regulate the IgE response and protective immunity in mice. Exp. Parasitol. 73, 500-511 (1991).

15. Morris, S. C. et al. Endogenously produced IL-4 nonredundantly stimulates CD8 +T cell proliferation. J. Immunol. 182, 1429-1438 (2009).

16. Pedras-Vasconcelos, J. A. \& Pearce, E. J. Type 1 CD $8+T$ cell responses during infection with the helminth Schistosoma mansoni. J. Immunol. 157, 3046-3053 (1996).

17. Akue, A. D., Lee, J. Y. \& Jameson, S. C. Derivation and maintenance of virtual memory CD8 T cells. J. Immunol. 188, 2516-2523 (2012).

18. Haluszczak, $C$. et al. The antigen-specific $C D 8+T$ cell repertoire in unimmunized mice includes memory phenotype cells bearing markers of homeostatic expansion. J. Exp. Med. 206, 435-448 (2009).
19. Hamilton, S. E., Wolkers, M. C., Schoenberger, S. P. \& Jameson, S. C. The generation of protective memory-like $\mathrm{CD} 8+\mathrm{T}$ cells during homeostatic proliferation requires CD4+T cells. Nat. Immunol. 7, 475-481 (2006).

20. Lee, J. Y., Hamilton, S. E., Akue, A. D., Hogquist, K. A. \& Jameson, S. C. Virtual memory CD8 T cells display unique functional properties. Proc. Natl. Acad. Sci. USA 110, 13498-13503 (2013).

21. Jameson, S. C., Lee, Y. J. \& Hogquist, K. A. Innate memory T cells. Adv. Immunol. 126, 173-213 (2015).

22. Lee, Y. J., Jameson, S. C. \& Hogquist, K. A. Alternative memory in the CD8 T cell lineage. Trends Immunol. 32, 50-56 (2011).

23. Lee, Y. J., Holzapfel, K. L., Zhu, J., Jameson, S. C. \& Hogquist, K. A. Steady-state production of IL- 4 modulates immunity in mouse strains and is determined by lineage diversity of iNKT cells. Nat. Immunol. 14, 1146-1154 (2013).

24. Weinreich, M. A., Odumade, O. A., Jameson, S. C. \& Hogquist, K. A. T cells expressing the transcription factor PLZF regulate the development of memorylike CD8+T cells. Nat. Immunol. 11, 709-716 (2010).

25. Sosinowski, T. et al. CD8alpha+dendritic cell trans presentation of IL-15 to naive $\mathrm{CD} 8+\mathrm{T}$ cells produces antigen-inexperienced $\mathrm{T}$ cells in the periphery with memory phenotype and function. J. Immunol. 190, 1936-1947 (2013).

26. White, J. T. et al. Virtual memory T cells develop and mediate bystander protective immunity in an IL-15-dependent manner. Nat. Commun. 7, 11291 (2016).

27. King, I. L. et al. The mechanism of splenic invariant NKT cell activation dictates localization in vivo. J. Immunol. 191, 572-582 (2013).

28. Berg, R. E., Crossley, E., Murray, S. \& Forman, J. Memory CD8+T cells provide innate immune protection against Listeria monocytogenes in the absence of cognate antigen. J. Exp. Med. 198, 1583-1593 (2003).

29. Boyman, O., Kovar, M., Rubinstein, M. P., Surh, C. D. \& Sprent, J. Selective stimulation of $\mathrm{T}$ cell subsets with antibody-cytokine immune complexes. Science 311, 1924-1927 (2006).

30. Morrot, A., Hafalla, J. C., Cockburn, I. A., Carvalho, L. H. \& Zavala, F. IL-4 receptor expression on CD8+T cells is required for the development of protective memory responses against liver stages of malaria parasites. J. Exp. Med. 202, 551-560 (2005).

31. Kurzweil, V., LaRoche, A. \& Oliver, P. M. Increased peripheral IL-4 leads to an expanded virtual memory CD8+population. J. Immunol. 192, 5643-5651 (2014).

32. LaPorte, S. L. et al. Molecular and structural basis of cytokine receptor pleiotropy in the interleukin-4/13 system. Cell 132, 259-272 (2008).

33. Mohrs, M. et al. Differences between IL-4- and IL-4 receptor alpha-deficient mice in chronic leishmaniasis reveal a protective role for IL-13 receptor signaling. J. Immunol. 162, 7302-7308 (1999).

34. Nelms, K., Keegan, A. D., Zamorano, J., Ryan, J. J. \& Paul, W. E. The IL-4 receptor: signaling mechanisms and biologic functions. Annu. Rev. Immunol. 17, 701-738 (1999).

35. Newcomb, D. C. et al. A functional IL-13 receptor is expressed on polarized murine CD4+Th17 cells and IL-13 signaling attenuates Th17 cytokine production. J. Immunol. 182, 5317-5321 (2009).

36. Park, H. J. et al. Effect of IL-4 on the development and function of memory-like CD8 T cells in the peripheral lymphoid tissues. Immune Netw. 16, 126-133 (2016).

37. Tripathi, P. et al. IL-4 and IL-15 promotion of virtual memory CD8+T cells is determined by genetic background. Eur. J. Immunol. 46, 2333-2339 (2016).

38. Renkema, K. R. et al. IL-4 sensitivity shapes the peripheral CD8+T cell pool and response to infection. J. Exp. Med. 213, 1319-1329 (2016).

39. Sutherland, R. E. et al. Parasitic infection improves survival from septic peritonitis by enhancing mast cell responses to bacteria in mice. PLoS One 6, e27564 (2011).

40. du Plessis, N. et al. Acute helminth infection enhances early macrophage mediated control of mycobacterial infection. Mucosal Immunol. 6, 931-941 (2013).

41. Erb, K. J., Trujillo, C., Fugate, M. \& Moll, H. Infection with the helminth Nippostrongylus brasiliensis does not interfere with efficient elimination of Mycobacterium bovis BCG from the lungs of mice. Clin. Diagn. Lab. Immunol. 9, 727-730 (2002).

42. Rafi, W., Bhatt, K., Gause, W. C. \& Salgame, P. Neither primary nor memory immunity to Mycobacterium tuberculosis infection is compromised in mice with chronic enteric helminth infection. Infect. Immun. 83, 1217-1223 (2015).

43. Tao, L. \& Reese, T. A. Making mouse models that reflect human immune responses. Trends Immunol. 38, 181-193 (2017).

44. Masopust, D., Sivula, C. P. \& Jameson, S. C. Of mice, dirty mice, and men: using mice to understand human immunology. J. Immunol. 199, 383-388 (2017).

45. Mohrs, K., Wakil, A. E., Killeen, N., Locksley, R. M. \& Mohrs, M. A two-step process for cytokine production revealed by IL-4 dual-reporter mice. Immunity 23, 419-429 (2005).

46. Mohrs, M., Shinkai, K., Mohrs, K. \& Locksley, R. M. Analysis of type 2 immunity in vivo with a bicistronic IL-4 reporter. Immunity 15, 303-311 (2001).

47. Mullarky, I. K. et al. Infection-stimulated fibrin deposition controls hemorrhage and limits hepatic bacterial growth during listeriosis. Infect. Immun. 73, 3888-3895 (2005).

48. Szaba, F. M. et al. TNFalpha and IFNgamma but not perforin are critical for CD8 T cell-mediated protection against pulmonary Yersinia pestis infection. PLoS Pathog. 10, e1004142 (2014). 